Teil III Einzelfragen 


\title{
Die Vertragserrichtung im Internet in der Volksrepublik China
}

\author{
Anne Sophie Ortmanns
}

\section{Einleitung}

Jenseits geografischer Grenzen bilden elektronische Verträge die rechtliche Grundlage für den in der Volksrepublik China florierenden E-Commerce-Markt ${ }^{1}$ und der damit einhergehenden Vernetzung der Gesellschaft. Der chinesische Gesetzgeber hat diese Entwicklung nicht erst seit Erlass des am 28.5.2020 verabschiedeten und am 1.1.2021 in Kraft getretenen Zivilgesetzbuches der Volksrepublik China $(\mathrm{ZGB})^{2}$ im Blick und in den letzten Jahren immer wieder mit dem Erlass von Rechtsnormen auf sie reagiert. Da im Internet geschlossene Verträge sich von herkömmlichen im Wesentlichen nur durch den Einsatz von Fernkommunikationsmitteln und die dadurch entstehende Anonymität der Parteien unterscheiden, liegen ihnen neben speziellen auch die allgemeinen, den Vertragsschluss betreffenden Vorschriften zugrunde, die in dem folgenden Beitrag somit ebenfalls Berücksichtigung finden. Ein besonderes Augenmerk liegt neben den Vorschriften des ZGB auf jenen des am 31.8.2018 verabschiedeten und am 1.1.2019 in Kraft getretenen Gesetzes über den E-Commerce der Volksrepublik China (ECG) ${ }^{3}$, die der Gesetzgeber bei der Kodifizierung des ZGB teils übernommen hat.

In einem ersten Schritt erfolgt eine grundlegende begriffliche Klärung der für die Thematik relevanten Terminologie. Es folgen Ausführungen über die in ZGB und ECG geschaffenen Regelungen über den Errichtungszeitpunkt im Internet geschlossener Verträge sowie das Verhältnis

1 Mit einem Umsatz von 1.343,5 Milliarden US-Dollar im Jahr 2020 ist die VR China der weltweit führende E-Commerce-Markt. Eine Fortsetzung dieses Trends in den kommenden Jahren ist zu erwarten, vgl. hierzu den E-Commerce-Report der Statistikdatenbank statista, https://www.statista.com/study/42335/ecommerce-r eport/>, zuletzt eingesehen am 2.8.2021.

2 Übersetzung ins Deutsche von DING Yijie/Peter Leibküchler/Nils Klages/Knut Benjamin Pißler, in: Zeitschrift für Chinesisches Recht 2020, Nr. 3-4, S. $207 \mathrm{ff}$.

3 Übersetzung ins Deutsche von Anne Sophie Ortmanns, in: Zeitschrift für Chinesisches Recht 2020, Nr. 2, S. 153 ff. 
dieser Regelungen zueinander. Anschließend wird der Zugang elektronischer Willenserklärungen als gängige Voraussetzung für die Vertragserrichtung im Internet beleuchtet. Dargestellt werden die Zugangskriterien sowie die Verteilung des Übermittlungsrisikos bei dem Austausch elektronischer Willenserklärungen. Hierauf folgen Ausführungen zu allgemeinen Geschäftsbedingungen (AGB), die untrennbar mit spezifischen digitalen Errichtungsformen verbunden sind, insbesondere sogenannten Click- und Browse-Wrap-Verträgen. Im Fokus stehen dabei die vor dem Hintergrund der Informationsasymmetrie zwischen AGB-Verwender und Vertragspartner geschaffenen Hinweis- und Erklärungspflichten des AGB-Verwenders, die im E-Commerce aufgrund der fehlenden haptischen Wahrnehmbarkeit der Vertragsgegenstände von besonderer Bedeutung sind. Sodann wird untersucht, welche rechtlichen Folgen sich aus dem praktisch relevanten Fall ergeben, dass Preise auf einer E-Commerce-Plattform falsch ausgewiesen werden. Anschließend wird eine Einordnung der elektronischen Form in das Gefüge zivilrechtlicher Formerfordernisse vorgenommen, bevor abschließend ein wertendes Resümee der herausgearbeiteten Ergebnisse erfolgt.

Da es sich bei den in diesem Beitrag im Fokus stehenden Vorschriften überwiegend um noch junge Regelungen handelt, die weder im Schrifttum noch in der Rechtsprechung eine umfassende Aufarbeitung erfahren haben, kann dieser Beitrag dem Rechtsanwender keine die Thematik erschöpfende und insofern vollends verlässliche Orientierungshilfe bieten. Vielmehr liegt das Ziel in der Erschließung und Auswertung bestehender Quellen als Momentaufnahme für den deutschsprachigen Leser, deren Gestalt sich in Zukunft durch weitere Entwicklungen in Rechtsprechung und Literatur mutmaßlich noch wandeln wird.

\section{Der Vertragsschluss im Internet in der Volksrepublik China}

Eine eindeutige gesetzliche Definition des elektronischen Vertrages existiert im chinesischen Recht nicht. ${ }^{4}$ Im Schrifttum werden elektronische Verträge definiert als Verträge, die von Parteien durch das Internet im We-

4 XU Dongjin (许东瑾), Bestimmung der Zivilgeschäftsfähigkeit Minderjähriger zum Abschluss elektronischer Verträge (未成年人订立电子合同的民事行为能力 认定), Modern Economic Information (现代经济信息) 2020, Nr. 1, S. 152, 152; LÜ Zushan (吕组善)/PENG Sen (彭森)/YIN Zhongqing (尹中卿) (Hrsg.), Erläuterung des E-Commerce-Gesetzes (中国人民共和国电子商务法条文释义), Peking, 2018, S. 142 . 
ge eines elektronischen Datenaustausches (EDI) oder elektronischer Post (E-Mail) geschlossen werden. ${ }^{5} \mathrm{Da}$ im Internet errichtete Verträge sich von herkömmlichen mithin lediglich durch den Einsatz von Fernkommunikationsmitteln unterscheiden, gelten die grundlegenden Begriffsbestimmungen des ZGB über Verträge auch insoweit. ${ }^{6}$ Definiert wird ein Vertrag in $\$ 464$ ZGB als eine rechtliche Vereinbarung, die zwischen Zivilrechtssubjekten getroffen, geändert und beendet wird. ${ }^{7}$ Gemäß $₫ 471$ ZGB können die Parteien Vertragsbeziehungen im Wege des Austausches von Angebot und Annahme oder auf anderem Wege eingehen. ${ }^{8}$ Gegenüber $\mathbb{} 13$ Vertragsgesetz (VG) ${ }^{9}$ wurde der Passus ,auf anderem Wege“ neu hinzugefügt. Auf anderem Wege geschlossene Verträge sind unter anderem elektronische Verträge. ${ }^{10}$

\section{Errichtungszeitpunkt nach $\$ 491$ Abs. 2 ZGB und $\$ 49$ Abs. 1 ECG}

Während ohne eine vertragliche Grundlage nur ein auf Ersatz des negativen Interesses gerichteter Anspruch aus culpa in contrahendo ${ }^{11}$ oder deliktische Ansprüche greifen, zieht die Bindung an einen Vertrag weitreichendere Rechtsfolgen nach sich, und die Frage nach dem Errichtungszeitpunkt

5 WANG Liming (王利明), Studie über das E-Commerce-Recht (电子商务法研究), Peking, 2003, S. 77; ZHENG Ning (郑宁), Einfluss und Reaktion des Zivilgesetzbuches auf die Medienbranche (民法典对传媒行业的影响及应对), China Publishing Journal (中国出版) 2020, Nr. 17, S. 5, 9.

6 Kommentierung des OVG zum Vertragsrechtsbuch des ZGB, Kleine Führungsgruppe zur Implementierungsarbeit des Zivilgesetzbuches des OVG (Hrsg.) (最高 人民法院民法典贯彻实施工作领导小组主编), Verständnis und Anwendung des Zivilgesetzbuches (中华人民共和国民法典理解与适用), Peking 2020, S. 206.

7 Dies entspricht im Wesentlichen $\$ 2$ Vertragsgesetz (VG), in dem Zivilsubjekte konkret als Bürger, juristische Personen und andere Organisationen definiert werden. Gemäß $\$ 1260$ ZGB wurde das Vertragsgesetz mit Inkrafttreten des ZGB aufgehoben.

8 In $\$ 13$ VG heißt es: „Zur Vertragserrichtung verwenden die Parteien Angebot und Annahme."

9 Übersetzung ins Deutsche von Frank Münzel, Chinas Recht 15.3.1999/1.

10 Knut Benjamin Pißler, Das ZGB der VR China im Spiegel einer Kommentierung des OVG, in: Zeitschrift für Chinesisches Recht 2021, Nr. 2, S. 85, 93.

11 WANG Liming (王利明), Untersuchung des Vertragsrechts (合同法研究), Band 1, 2. Aufl. 2011, S. 366 ff., vgl. auch Yuanshi Bu, Genehmigungspflicht und zwingende Rechtsnormen im chinesischen Vertragsrecht, in: Recht der Internationalen Wirtschaft 2014, Nr. 9, S. 549, 550. 
ist insofern von zentraler Bedeutung. ${ }^{12}$ Nach $₫ 483$ ZGB kommt ein Vertrag mit Wirksamwerden der Annahme zustande, im Hinblick auf das $\mathrm{Zu}$ standekommen elektronischer Verträge sind darüber hinaus die $\$ \mathbb{S} 491$ Abs. 2 ZGB und $\$ 49$ Abs. 1 ECG hinsichtlich des Errichtungszeitpunktes zu berücksichtigen. Gemäß $₫ 49$ Abs. 1 ECG ist ein Vertrag errichtet, wenn die durch die E-Commerce-Betreiber bekannt gemachten Informationen über Waren oder Dienstleistungen den Bedingungen für ein Angebot entsprechen und ein Nutzer die Ware oder Dienstleistung ausgewählt und seine Bestellung erfolgreich abgegeben hat. Die Parteien können hiervon gemäß $₫ 49$ Abs. 1 S. 2 ECG abweichende Vereinbarungen treffen. Diese Regelungen hat der Gesetzgeber in $\$ 491$ Abs. 2 ZGB aufgenommen ${ }^{13}$ und die Frage nach einer vorrangigen Anwendung des $\$ 49$ Abs. 1 ECG als lex specialis erübrigt sich insofern. ${ }^{14}$ Diese Frage stellte sich in Bezug auf die Entwurfsfassung, deren $₫ 283$ Abs. 2 ZGB-E anders als $₫ 491$ ZGB noch vorsah, dass ein abweichender Zeitpunkt des Vertragsschlusses nach der Verkehrssitte erlaubt sein sollte. ${ }^{15}$ Der Gesetzgeber entschied sich für die geltende Fassung, weil er befürchtete, dass andernfalls beispielsweise der Zeitpunkt des Zugangs der Bestellbestätigung in der Praxis als Verkehrssitte hätte angesehen und dies dem verbraucherschützenden Hintergrund des $\$ 49$ Abs. 1 ECG hätte zuwiderlaufen können. ${ }^{16}$

Mit der Schaffung des $₫ 49$ Abs. 1 ECG und dessen Übernahme in $\$ 491$ Abs. 2 ZGB hat der Gesetzgeber die für den Zeitpunkt des Zustandekommens des elektronischen Vertrages zentrale Frage geregelt, zu welchem

12 So auch SUN Liangguo (孙良国), Bestimmung des Errichtungszeitpunkts von Verträgen und Werturteil des Vertragsrechts am Beispiel von XIA Wei gegen Amazon Excellence wegen der unbefugten Löschung einer Bestellung (合同成立时点的 确定与合同法的价值判断一以 “夏伟诉亚马逊卓越擅自删除订单案”为例), ECUPL Journal (华东政法大学学报) 2018, Nr. 2, S. 120, 124.

13 Die Definition des ZGB unterscheidet sich aber insoweit von der des ECG, als das ZGB keine Bereichsausnahme wie die des $\$ 2$ Abs. 3 S. 2 ECG vorsieht. Diese gilt für Finanzprodukte und -dienstleistungen sowie Dienstleistungen wie jene in Bezug auf Nachrichten, Audio- und Videoprogramme, Verlags- und Kulturprodukte, die durch Informationsnetzwerke zur Verfügung gestellt werden.

14 Aus $\$ 92$ Gesetzgebungsgesetz der VR China, chinesisch-deutsch in: Zeitschrift für Chinesisches Recht 2015, Nr. 3, S. 259 ff., ergibt sich, dass der Grundsatz lex specialis derogat legi generali grundsätzlich auch im chinesischen Recht gilt. Siehe hierzu auch Yuanshi Bu, Normenkollisionen und Normenkontrolle in China, in: Recht der Internationalen Wirtschaft 2015, Nr. 12, S. 781, 786.

15 Yuanshi Bu, Hintergrund, Bestandsaufnahme und Anmerkungen zum BT ZGB mit dem Vertrags- und Erbrecht im Fokus, in: Yuanshi Bu (Hrsg.), Der Besondere Teil der chinesischen Zivilrechtskodifikation, Tübingen 2019, S. 12.

16 Yuanshi Bu (Fn. 15), S. 12 m.w.N. 
Zeitpunkt das reziproke Einverständnis der Parteien als gegeben angesehen wird, die Parteien mithin an den Vertrag gebunden sind. ${ }^{17}$ Im Schrifttum wird der Hintergrund der Regelung des $\$ 49$ Abs. 1 ECG und des Vertragsschlusses im Zeitpunkt der Abgabe der Bestellung in der Orientierung des Gesetzgebers an tatsächlichen Gegebenheiten gesehen. So bestehe auf E-Commerce-Plattformen wie Jingdong die Möglichkeit, einen Artikel mehrere Tage lang in einem digitalen Warenkorb zu platzieren, ohne dass der Kunde zu Vertragsschluss oder Zahlung aufgefordert wird, und bis zur Aufgabe der Bestellung könne dieser Vorgang durch beide Seiten ohne Weiteres technisch rückgängig gemacht werden. ${ }^{18}$

a) Die Bedingungen für ein Angebot i.S.v. $\$ 491$ Abs. 2 ZGB und $\$ 49$ Abs. 1 ECG

Vor Erlass des $\$ 49$ Abs. 1 ECG und des $\$ 491$ Abs. 2 ZGB war umstritten, ob die Präsentation einer Ware oder Dienstleistung auf einer E-CommercePlattform als Angebot oder als invitatio ad offerendum zu klassifizieren war. ${ }^{19}$ In der Regel wurde sie als Aufforderung zur Abgabe eines Angebotes, mithin als invitatio ad offerendum i.S.v. $\$ 15$ S. $1 \mathrm{VG}^{20}$ gesehen. ${ }^{21}$ Ein Angebot lag sodann in der Aufgabe der Bestellung, und die Bestellbestätigung, die der auf der E-Commerce-Plattform tätige Betreiber anschließend versendet, enthielt in der Regel die Klarstellung, dass dieser in dem Stadium noch nicht gebunden sei und die Annahme erst durch Zusendung der Versandbestätigung erfolge. ${ }^{22}$ In der Bestellbestätigung lag nur ausnahmsweise eine Annahme, so beispielsweise wenn es in ihr hieß, die Ware wer-

17 XUE Jun (薛军), Analyse der Probleme bei der Errichtung elektronischer Verträge (电子合同成立问题探析), Journal of Law Application (法律适用) 2021, Nr. 3, S. $25,26$.

18 WANG Hongliang (王洪亮), Analyse und Einschätzung sowie Struktur der Regelungen zur Errichtung elektronischer Verträge (电子合同订立新规则的评析与构 建), Law Science Magazine (法学杂志) 2018, Nr. 4, S. 32, 36.

19 Urteil des Dritten Mittleren Volksgerichts Beijing über die vorvertragliche Haftung der Beijing Century Excellence Information Technology Co., Ltd. gegenüber LI Shanghui aufgrund einer falschen Preisauszeichnung, (2017) Jing 03 Min Zhong Nr. 1140.

20 Diese Regelung entspricht der des $₫ 473$ S. 1 ZGB.

21 Siehe nur WU Jinyu (吴瑾瑜), Über die rechtlichen Auswirkungen falsch ausgewiesener Preise auf Internetseiten (论网站标价错误之法律效力), The Taiwan Law Review (月旦法学杂志) 2010, Nr. 1, S. 39, 55.

22 WANG Hongliang (Fn. 18), S. 37. 
de so schnell wie möglich versendet oder die Lieferung sei bereits vorbereitet. ${ }^{23}$ Seit Inkrafttreten der Neuregelungen der $\$ \mathbb{S} 49$ Abs. 1 ECG und 491 Abs. 2 ZGB, die den Zeitpunkt der Vertragserrichtung explizit für den Zeitpunkt der Abgabe der Bestellung vorsehen, stellt sich fortan die Frage, wann Informationen, die auf einer Webseite präsentiert werden, ein Angebot im Rechtssinne darstellen. ${ }^{24}$ Hierzu ist auf die gesetzliche Definition des Angebots in $₫ 472$ ZGB abzustellen, demzufolge ein Angebot eine Willenserklärung ist, die in der Absicht abgegeben wird, mit einem anderen einen Vertrag zu errichten. ${ }^{25}$ Der Inhalt des Angebotes muss gemäß $\$ 472$ Nr. 1 ZGB konkret festgelegt sein und drückt aus, dass mit der Annahme durch den Empfänger des Angebots der Anbietende an seine Willenserklärung gebunden wird. Ob der Inhalt konkret i.S.v. $\$ 472 \mathrm{Nr}$. 1 ZGB festgelegt ist, sei bei im Internet errichteten Verträgen anhand der für den ECommerce spezifischen Charakteristika zu bestimmen. Dabei seien auch neuere Erscheinungsformen wie Live-Streamings zu berücksichtigen, unabhängig von der Art der Informationsvermittlung sei es jedoch in jedem Fall notwendig, die Gesamtschau der während der Veranstaltung präsentierten Inhalte ins Blickfeld zu nehmen. Hinsichtlich der Frage, welche Inhalte ein Vertrag in der Regel enthält, wird ferner auf $\$ 470$ ZGB rekurriert, der indes lediglich eine Orientierungshilfe biete und eine Gesamtbe-

23 WANG Hongliang (Fn. 18), S. 38. Für eine ausführliche Darstellung des Streits siehe SUN Liangguo (Fn. 12), S. 122 ff., der sich für den Zeitpunkt der Vertragserrichtung bei Versendung der Ware ausspricht und kritisiert, dass eine differenzierte Interessenabwägung in Bezug auf den Zeitpunkt der Vertragserrichtung in der Rechtsprechung oftmals ausbleibe. Beispielhaft sei hierfür das Urteil Xia Wei gegen Amazon Excellence des Volksgerichtes Chaoyang, Beijing, (2013) Chao Min Chu Zi Nr. 2145, in dem das Gericht davon ausging, dass der Vertrag trotz der AGB von Amazon über die Zusendung der Versandbestätigung als Errichtungszeitpunkt bereits im Zeitpunkt der Bestellung des Käufers zustande gekommen sei. Amazon sei der Hinweispflicht nicht in hinreichender Weise nachgekommen, da die AGB nur durch das Anklicken eines nicht leicht erkennbaren Links für Verbraucher einsehbar gewesen seien. Bei der Überprüfung der Bestellung habe Amazon Informationen über das Produkt, die Bestellmenge, die Lieferadresse und die Zahlungsmethode in fettgedruckter Schrift angezeigt, die AGB jedoch nur in normaler, farblich schlecht gesättigter Schrift am unteren Seitenrand.

24 Vgl. LÜ Zushan/PENG Sen/YIN Zhongqing (Hrsg.) (Fn. 4), S. 148 f., die es ablehnen, auf Internetseiten präsentierte Waren oder Dienstleistungen nunmehr kategorisch als Angebot zu klassifizieren. Vielmehr liege die entscheidende Frage nun darin, wann die Präsentation die Bedingungen für ein Angebot i.S.v. $\$ 49$ Abs. 1 S. 1 ECG erfüllt.

25 Kommentierung des OVG zum Vertragsrechtsbuch des ZGB (Fn. 6), S. 206. 
trachtung der präsentierten Waren und Dienstleistungen nicht ersetze. ${ }^{26}$ Die Formulierung in $\$ 472 \mathrm{Nr} .1 \mathrm{ZGB}$, der Inhalt sei bestimmt, sei so zu verstehen, dass der Inhalt bestimmbar sein muss. Eine Vertragspartei sei konkret und bestimmt i.S.v. $\$ 472 \mathrm{Nr} .1 \mathrm{ZGB}$, wenn sie mit entsprechenden Hintergrundinformationen identifizierbar sei. Dies werde besonders bei Hybridplattformen relevant, die sowohl durch den Plattformbetreiber selbst betrieben als auch durch Drittanbieter genutzt werden. Der Vertragsgegenstand und Preis seien konkret und bestimmt i.S.v. $\mathbb{} 472 \mathrm{Nr} .1$ ZGB, wenn es ein objektives und ex ante klar festgelegtes Kriterium oder eine solche Regel zur Bestimmung gebe. ${ }^{27}$

Für die Frage, wann die Bedingungen für ein Angebot erfüllt sind, ist ferner der angesichts seiner inhaltlichen Zirkularität ${ }^{28}$ allerdings wenig aussagekräftige $\mathbb{4} 472 \mathrm{Nr} .2$ ZGB relevant, der voraussetzt, dass der Anbietende in dem Angebot deutlich macht, mit der Annahme durch den Empfänger des Angebots an diese Willenserklärung gebunden zu sein. Was die konkrete Form betrifft, so kann das „Deutlichmachen“, das in $₫ 472 \mathrm{Nr} .2$ ZGB genannt wird, ausdrücklich oder stillschweigend erfolgen. ${ }^{29}$

\section{b) Möglichkeit der abweichenden Vereinbarung des $\$ 491$ Abs. 2 a. E. ZGB und \49 Abs. 1 S. 2 ECG}

Indem der Gesetzgeber den Parteien die Möglichkeit der abweichenden Vereinbarung einräumt, trägt er der Privatautonomie Rechnung. ${ }^{30}$ Wenn

26 XUE Jun (Fn. 17), S. 26; LÜ Zushan/PENG Sen/YIN Zhongqing (Hrsg.) (Fn. 4), S. 148 f., verweisen in Bezug auf $\$ 49$ Abs. 1 ECG ebenfalls auf die $\$ \$ 14 \mathrm{ff}$. VG, die im Einzelnen festlegen, wann ein Angebot und wann eine invitatio ad offerendum vorliegen. Hervorgehoben wird allerdings auch, dass im E-Commerce-Gesetz diesbezüglich gerade keine genaue Aussage getroffen wird und die Frage, ob ein Angebot vorliegt, nach den Umständen des Einzelfalles zu entscheiden sei. Es liege hierbei an der Rechtsprechung, bei der Entscheidung von Einzelfällen allgemeingültige Kriterien für die Beantwortung dieser Frage zu entwickeln.

27 XUE Jun (Fn. 17), S. 27.

28 So auch XUE Jun (Fn. 17), S. 27.

29 XUE Jun (Fn. 17), S. 27. Erfolge das Deutlichmachen stillschweigend, müsse aus den präsentierten Informationen vernünftigerweise abgeleitet werden können, dass es sich um ein Angebot im Rechtssinne handelt. Dabei sei der Beurteilungsmaßstab eines objektiven Dritten zugrunde zu legen, die Erklärung müsse mithin nach der allgemeinen gesellschaftlichen Auffassung vernünftigerweise als Angebot verstanden werden können.

30 Kommentierung des OVG zum Vertragsrechtsbuch des ZGB (Fn. 6), S. 206. XUE Jun (Fn. 17), S. 27. Die Privatautonomie wird in der Zwischenzeit als legitimes 
die Parteien mithin objektiv so handeln, als würden sie ein Angebot abgeben, sie aber durch besondere Vereinbarung ausdrücklich ausschließen, dass ihr Verhalten als rechtliches Angebot definiert wird, wird aus dem rechtlichen Verhalten grundsätzlich eine unverbindliche invitatio ad offerendum. ${ }^{31}$ In diesem Fall stellt in der Regel die Abgabe der Bestellung ein verbindliches Angebot dar, vorrangig gilt aber auch insoweit die Vereinbarung der Parteien. ${ }^{32}$ Die Möglichkeit der abweichenden Vereinbarung über den Errichtungszeitpunkt ist angesichts der Quantität der im Internet durch ein- und denselben Händler abgeschlossenen Verträge und der Tatsache, dass es den Parteien vor dem Zustandekommen des Vertrages in diesen Fällen in der Regel nicht möglich sein wird, sich darüber zu verständigen, ob ihre Handlungen ein Angebot oder eine invitatio ad offerendum darstellen, allerdings praktisch überwiegend dann von Bedeutung, wenn die Möglichkeit abweichender Vereinbarungen jene in Form von AGB einschließt. ${ }^{33}$ Neben diesem eher praktischen Argument spricht für die Möglichkeit der Vereinbarung durch AGB zudem der Gesetzeswortlaut, der keine besondere Formulierung für „individualvertraglich“ (个别商议的) enthält.

c) $\$ 49$ Abs. 2 ECG

Die während des Gesetzgebungsverfahrens des ECG sehr umstrittene ${ }^{34}$ Vorschrift des $₫ 49$ Abs. 2 ECG hat im Gegensatz zu $₫ 49$ Abs. 1 ECG keinen Niederschlag in der Zivilrechtskodifikation gefunden. $\$ 49$ Abs. 2 ECG sieht vor, dass E-Commerce-Betreiber nicht durch Geschäftsbedin-

gesetzgeberisches Ziel angesehen, während sie zu Zeiten der Kulturrevolution noch als Symbol des Kapitalismus abgelehnt wurde, vgl. Jörg Binding/Sophia Kurz, Formularklauseln im chinesischen Zivilrecht, in: Recht der Internationalen Wirtschaft 2013, Nr. 7, S. 424, 425. Teils wird vertreten, eine abweichende Vereinbarung über den Errichtungszeitpunkt sei der Natur der Sache nach gar nicht möglich, da ein Vertrag immer im Zeitpunkt der Einigung zustande komme und die Parteien hierüber nichts Abweichendes vereinbaren könnten, vgl. SUN Liangguo (Fn. 12), S. 128.

31 XUE Jun (Fn. 17), S. 27.

32 XUE Jun (Fn. 17), S. 27.

33 Dafür, dass abweichende Vereinbarungen jene in Gestalt von AGB umfassen, vgl. die Kommentierung des OVG zum Vertragsrechtsbuch des ZGB (Fn. 6), S. 207; XUE Jun (Fn. 17), S. 27; LÜ Zushan/PENG Sen/YIN Zhongqing (Hrsg.) (Fn. 4), S. 149.

34 XUE Jun (Fn. 17), S. 29. 
gungen oder in anderer Form vereinbaren dürfen, dass ein Vertrag nach Zahlung durch einen Verbraucher nicht zustande kommt; falls Geschäftsbedingungen etc. einen solchen Inhalt enthalten, ist dieser unwirksam. Legislativer Beweggrund für den Erlass dieser Vorschrift war im Wesentlichen die Eindämmung des Phänomens, dass E-Commerce-Betreiber Verbraucher dazu verleiten, ihre Waren oder Dienstleistungen zu einem vergünstigten Preis zu bestellen und ihren Antrag sodann stornieren, wodurch Käufern Möglichkeiten des Erwerbs bei anderen Betreibern im Rahmen groß angelegter Werbeaktionen und E-Commerce-Betreibern Handelsmöglichkeiten entgehen. ${ }^{35}$ Anders als zum Teil in der Rechtsprechung heißt es in der Literatur, $\$ 49$ Abs. 2 ECG mache inhaltlich wenig Sinn, lege man die Vorschrift wörtlich aus und unterstelle, dass sie Fälle erfasst, in denen die Parteien im Wege von AGB vereinbart haben, dass ein Vertrag nicht zustande kommt, nachdem ein Verbraucher seine Zahlung geleistet hat. ${ }^{36}$ Daher wird die Vorschrift teleologisch dahingehend ausgelegt, dass sie Vereinbarungen darüber erfasst, was ein Angebot und was eine invitatio ad offerendum darstellt. ${ }^{37}$ Gleichwohl werde auch bei Zugrundelegung dieses Verständnisses das dogmatische Grundgerüst des Vertrages erschüttert, das auf der Annahme einer Trennung der Stadien der Vertragserrichtung und Vertragserfüllung fuße. Entscheidend für die Frage, ob ein Vertrag geschlossen ist, könne lediglich die Existenz einer Vereinbarung zwischen den Parteien sein, nicht aber die Frage, ob eine Partei ihre vertraglich geschuldete Leistung erbracht hat oder nicht. ${ }^{38}$

Aus praktischer Perspektive wird ferner hervorgehoben, dass die Verknüpfung der Vertragserrichtung und Vertragserfüllung in $\$ 49$ Abs. 2 ECG im Hinblick auf spezielle Geschäftsmodelle Probleme verursache.

35 XUE Jun (Fn. 17), S. 29. Im Chinesischen ist die Rede von 砍单, wörtlich: „Abschlagen der Bestellung“.

36 So auch XUE Jun (Fn. 17), S. 29. Wörtlich und ohne lange Erläuterung wendet die Vorschrift aber beispielsweise das Internetgericht Beijing in seinem Urteil vom 5.3.2021 an, (2020) Jing 0491 Min Chu Nr. 24956.

37 XUE Jun (Fn. 17), S. 29, ähnlich LÜ Zushan/PENG Sen/YIN Zhongqing (Hrsg.) (Fn. 4) S. 150, die ohne genauere Erklärung unterstellen, dass $₫ 49$ Abs. 2 ECG die Frage regelt, ob E-Commerce-Betreiber und Verbraucher eine abweichende Vereinbarung über die Bindungswirkung der durch die Verbraucher abgegebenen Bestellung treffen können.

38 XUE Jun (Fn. 17), S. 29, hebt hervor, dass dem mit der Vorschrift anvisierten Ziel des Verbraucherschutzes durch andere Instrumentarien wie beispielsweise einer Einbeziehungskontrolle Rechnung zu tragen sei. Für die Unerheblichkeit der die Erfüllung des Vertrages betreffenden Fragen im Hinblick auf dessen Zustandekommen siehe auch SUN Liangguo (Fn. 12), S. 22. 
Dies gelte unter anderem für das Modell des sogenannten Gruppenkaufs (拼团模式), bei dem eine bestimmte Anzahl von Personen teilnehmen muss, damit die Verträge mit den Teilnehmern zu einem bestimmten Gruppenpreis zustande kommen. Dieses bei Verbrauchern durchaus beliebte Geschäftsmodell lasse sich vor dem Hintergrund des $\$ 49$ Abs. 2 ECG im Grunde nicht mehr verwirklichen. Ein ähnliches Problem stelle sich bei dem Vertragsmodell der sogenannten Blitzangebote (创购模式), bei denen ein Händler eine begrenzte Anzahl von Waren zu einem bestimmten vergünstigten Preis anbietet und die Interessenten den Kaufpreis im Voraus zahlen. Anschließend entscheide der Anbieter beispielsweise im Wege einer Auslosung, wer den Artikel zu einem vergünstigten Preis erhält. Nur mit dieser Person komme der Vertrag dann auch zustande. Praktisch zweifelhaft sei ferner, dass im Dunkeln bleibe, wann die Zahlung durch einen Verbraucher als erfolgt gilt. ${ }^{39}$ Wenn die Zahlung mithilfe eines Drittanbieters wie Alipay getätigt wird, stelle sich die Frage, ob der Eingang der Zahlung auf dem Alipay-Konto entscheidend ist oder die Zahlung erst berücksichtigt wird, nachdem der Käufer die Zahlung endgültig bestätigt hat.

Dem eingangs umrissenen Phänomen stornierter Anträge zu begegnen, indem die dem Vertragsschluss zugrunde liegende Logik geändert wird, erscheine daher fragwürdig und stelle einen nicht zu rechtfertigenden Eingriff in die Privatautonomie der Parteien dar. Dieser Eingriff sei auch nicht notwendig, da in diesen Fällen Schadensersatz nach den Grundsätzen der culpa in contrahendo zu leisten sei, gestützt darauf, dass der Abschluss des Vertrags als Vorwand genutzt wurde, um böswillig zu verhandeln, $\mathbb{} 500$ Nr. 1 ZGB. Es sei überdies Aufgabe des Plattformbetreibers und der Marktaufsichtsbehörden, dieses Phänomen durch Regulierungsmaßnahmen effektiv zu steuern. ${ }^{40}$ Es wird resümiert, dass die Vorschrift des $₫ 49$ Abs. 2 ECG rechtswissenschaftlich unhaltbar sowie wertungsmäßig unausgewogen und der Gesetzgeber mit der Schaffung des $₫ 49$ Abs. 2 ECG über das Ziel des Verbraucherschutzes daher weit hinausgeschossen sei. ${ }^{41}$

39 XUE Jun (Fn. 17), S. 29 f.

40 Vgl. XUE Jun (Fn. 17), S. 30

41 XUE Jun (Fn. 17), S. 30, a.A. LÜ Zushan/PENG Sen/YIN Zhongqing (Hrsg.) (Fn. 4), S. 150, die $\$ 49$ Abs. 2 ECG in den meisten Fällen für eine dem Verbraucherschutz zuträgliche Beschränkung der Privatautonomie des E-Commerce-Betreibers halten. 


\section{d) Verhältnis zwischen $\$ 491$ ZGB und $\$ 49$ Abs. 2 ECG}

Da bei der Kodifizierung des ZGB auf das ECG Bezug genommen wurde und $\$ 491$ Abs. 2 ZGB der Regelung des $\$ 49$ Abs. 1 ECG abgesehen von geringfügigen begrifflichen Unterschieden entspricht, stellt sich die Frage, ob der Gesetzgeber die terminologisch und inhaltlich fragwürdige Bestimmung des $\$ 49$ Abs. 2 ECG bewusst nicht in das ZGB aufgenommen hat oder es sich um ein Versehen handelt. Da es sich bei $₫ 49$ Abs. 1 und Abs. 2 ECG um eine Vorschrift handelt, erscheint unwahrscheinlich, dass dem Gesetzgeber ein Versehen unterlaufen ist. ${ }^{42}$ Neben der Tatsache, dass es sich bei der Kodifizierung des ZGB um einen Prozess des „Nehmens und Gebens" gehandelt habe, wird in der Literatur für diese Ansicht formaljuristisch vorgebracht, dass es sich bei dem ZGB um das jüngere Gesetz handele, dem daher der Vorrang einzuräumen sei. ${ }^{43}$ Konzediert wird indes, dass für die vorrangige Anwendung des ECG im Lichte des $\$ 11$ ZGB, der die vorrangige Anwendung spezieller Vorschriften regelt, der Charakter des $₫ 49$ Abs. 2 ECG als lex specialis gegenüber den Vorschriften des ZGB vorgebracht werden könne. ${ }^{44}$ Dies sei umso wichtiger, als es sich bei $\$ 49$ Abs. 2 ECG um eine speziell verbraucherschützende Vorschrift handele. Angesichts der Tatsache, dass die Kodifizierung des ZGB nicht darauf abziele, alle bisherigen Rechtsvorschriften in das ZGB aufzunehmen, sei es jedoch unangemessen, $\mathbb{\$} 11$ ZGB dahingehend auszulegen, alle Vorschriften in bestehenden Gesetzen, die nicht ausdrücklich aufgehoben wurden, als leges speciales im Verhältnis zum ZGB anzusehen. ${ }^{45}$ Diese Herangehensweise an die Rechtsanwendung würde das Potenzial für eine im Rahmen des Kodifizierungsprozesses stattfindende Erneuerung in signifikanter

42 So auch XUE Jun (Fn. 17), S. 31. Ferner ergibt sich aus der Kommentierung des OVG zum Vertragsrechtsbuch des ZGB, dass auch das OVG von der Anwendbarkeit der Vorschrift ausgeht (Fn. 6), S. 207.

43 XUE Jun (Fn. 17), S. $31 \mathrm{f}$.

44 Siehe zur Geltung des Grundsatzes lex specialis derogat legi generali schon oben, Fn. 14. Siehe zu dem Verhältnis zwischen ECG und ZGB im Allgemeinen auch LI Xiaocao (李小草), Untersuchung der Anwendung des Rechtssystems der ECommerce-Plattform zum Schutz des geistigen Eigentums gemäß dem E-Commerce-Gesetz (《电子商务法》电商平台知识产权保护规定的法体系适用研究), Journal of Law Application (法律适用) 2020, Nr. 13, S. 124 ff. Auch das OVG unterstellt in seiner Kommentierung zum Vertragsrechtsbuch des ZGB in Bezug auf $\$ 49$ Abs. 2 ECG dessen vorrangige Anwendbarkeit gegenüber dem ZGB als lex specialis (Fn. 6), S. 208.

45 XUE Jun (Fn. 17), S. 32. 
Weise verringern. ${ }^{46}$ Vielmehr sei im Einzelfall zu prüfen, ob der Grundsatz der vorrangigen Anwendung des spezielleren Gesetzes vor dem ZGB zu materiell gerechten Ergebnissen führt oder eine Anwendung der Regeln des ZGB zu einem erheblichen Interessenungleichgewicht führen würde. Nur wenn dies der Fall ist, sei $\$ 11$ ZGB einschlägig und dem spezielleren Gesetz der Vorrang einzuräumen. Angesichts der regelungstechnischen Probleme des $₫ 49$ Abs. 2 ECG und dem daraus resultierenden Interessenungleichgewicht sei davon auszugehen, dass der Gesetzgeber sich bei der Kodifizierung des ZGB bewusst gegen die Übernahme des $₫ 49$ Abs. 2 ECG entschieden habe. ${ }^{47}$

\section{e) Zwischenergebnis}

Hinsichtlich der zentralen Frage nach dem Errichtungszeitpunkt elektronischer Verträge und den in $\$ 491$ Abs. 2 ZGB und $\$ 49$ ECG insofern getroffenen Regelungen ist festzuhalten, dass in Übereinstimmung mit der Auffassung des Obersten Volksgerichts (OVG) neben der Anwendbarkeit der inhaltsgleichen $\$ \mathbb{} \$ 91$ Abs. 2 ZGB und 49 Abs. 1 ECG auch $\$ 49$ Abs. 2 ECG weiterhin praktisch relevant bleiben wird. Dies gilt, obgleich methodisch überzeugende Argumente gegen die Fortgeltung des $₫ 49$ Abs. 2 ECG sprechen.

\section{Zugang elektronischer Willenserklärungen}

Neben den Vorschriften über den Errichtungszeitpunkt ist für das Zustandekommen im Internet errichteter Verträge entscheidend, dass die von den Vertragsparteien abgegebenen Willenserklärungen ${ }^{48}$ einander zugehen. Das ZGB differenziert im Rahmen der empfangsbedürftigen Willenserklärungen insoweit zwischen Willenserklärungen, die in einem Gespräch abgegeben, und jenen, die nicht in einem Gespräch abgegeben wer-

46 Vgl. WANG Liming (王利明), Bei der korrekten Anwendung des Zivilgesetzbuches sind drei Arten von Beziehungen zu handhaben (正确适用民法典应处理好 三种关系), Modern Law Science (现代法学) 2020, Nr. 6, S. 3, 10.

47 XUE Jun (Fn. 17), S. 32.

48 Dass der Begriff der Willenserklärung explizit genannt und geregelt wird, wird als wesentlicher Fortschritt der Neuregelungen des ZGB gesehen, Yuanshi Bu, Die Kodifikation des chinesischen Zivilgesetzbuches - ausgewählte Fragen, in: Zeitschrift für Chinesisches Recht 2017, Nr. 3, S. 183, 191. 
den, vgl. $\$ 137$ Abs. 1 ZGB ${ }^{49}$. Relevant ist diese Unterscheidung auch im Hinblick auf den Zugang elektronischer Willenserklärungen. ${ }^{50}$ Eine Willenserklärung in Form eines elektronischen Datenschriftstücks ist eine nicht in einem Gespräch abgegebene Willenserklärung, vgl. $\mathbb{} 137$ Abs. 2 ZGB. ${ }^{51}$ Definiert werden elektronische Datenschriftstücke als Informationen, die auf elektronischem, optischem, magnetischem oder ähnlichem Wege erzeugt, gesendet, empfangen oder gespeichert werden und erst nach dem Übertragungsprozess sichtbar Gestalt annehmen. ${ }^{52}$

\section{a) Zugangskriterien}

Eine in einem Gespräch abgegebene Willenserklärung wird in dem Moment wirksam, in dem das Gegenüber von ihrem Inhalt Kenntnis erlangt, $\$ 137$ Abs. 1 ZGB, während eine nicht in einem Gespräch abgegebene Willenserklärung in dem Moment wirksam wird, in dem sie dem Gegenüber zugeht, $\mathbb{1} 137$ Abs. 2 S. 1 ZGB. Gesetzlich nicht definiert wird der Begriff des Zugangs. In der Literatur heißt es, der Zugang sei erfolgt, wenn die Willenserklärung so in den Machtbereich des Empfängers gelangt ist, dass dieser die Möglichkeit hat, von ihrem Inhalt Kenntnis zu erlangen. ${ }^{53}$ Die tatsächliche Kenntnisnahme durch den Empfänger sei keine Wirksamkeitsvoraussetzung, sodass dieser den Zeitpunkt des Zugangs einer bereits in seinem Machtbereich befindlichen Willenserklärung nicht durch vorsätzliches Nichtwissen kontrollieren könne. ${ }^{54} \mathrm{Da}$ Vertragsschlüsse im Internet in der Regel durch den Austausch nicht in einem Gespräch abgegebener Willenserklärungen erfolgen, muss die Annahme nach $₫ 481$ Abs. 2

49 Die Regelung des $₫ 137$ ZGB ersetzt ab Inkrafttreten des ZGB am 1.1.2021 die gleichlautende Vorschrift des $\$ 137$ Allgemeiner Teil des Zivilrechts der VR China, vgl. $1260 \mathrm{ZGB}$.

50 WANG Hongliang (Fn. 18), S. 33.

51 Vgl. hierzu auch die Übersicht von Yuanshi Bu (Fn. 48), S. 191.

52 WANG Hongliang (Fn. 18), S. 33.

53 SHEN Deyong (沈德咏) (Hrsg.), Verständnis und Anwendung des Allgemeinen Teils des Zivilrechts der Volksrepublik China (中华人民共和国民法总则条文理 解与适用(下)), Peking, 2017, S. 922; Yuanshi Bu, Chinese Civil Code - The General Part -, Baden-Baden 2019, S. 107 m.w.N.; dies entspricht der im deutschen Recht allgemein anerkannten Definition des Zugangs, nach der eine Willenserklärung dann zugegangen ist, wenn sie so in den Machtbereich des Empfängers gelangt ist, dass dieser die Möglichkeit zur Kenntnisnahme hat, MüKoBGB/Einsele, 9. Auflage 2021, $\$ 130$ Rn. 16 m.w.N.

54 WANG Hongliang (Fn. 18), S. 33. 
Nr. $2 Z^{2} G^{55}$ innerhalb einer vernünftigen Frist zugehen. Bei elektronischen Willenserklärungen wird hier regelmäßig von einer Frist von einem Tag ausgegangen. ${ }^{56}$

Speziell in Bezug auf elektronische Willenserklärungen wird in $\$ 137$ Abs. 2 ZGB wie in $₫ 16$ S. 2 VG danach differenziert, ob der Empfänger ein bestimmtes Computersystem zum Empfang elektronischer Willenserklärungen explizit benannt hat. $\mathbb{\$} 16 \mathrm{~S} .2 \mathrm{VG}$ sah indes noch vor, dass ein elektronisches Schriftstück als dem Empfänger zu der Zeit zugegangen gilt, zu der das Schriftstück erstmals in irgendein Computersystem des Empfängers gelangt. Diese Bestimmung wurde in der Literatur als unzureichend kritisiert, da sie voraussetze, dass der Empfänger seine E-Mails oder sein Computersystem ständig überprüfe und dem Erklärenden so der Raum genommen werde, von seiner Willenserklärung wieder Abstand zu nehmen. ${ }^{57} \mathrm{Im}$ Schrifttum wird dies als Hintergrund der Neuregelung des $\$ 137$ Abs. 2 S. $3 \mathrm{ZGB}^{58}$ gesehen, der nunmehr festlegt, dass die Willenserklärung vorbehaltlich einer abweichenden Vereinbarung der Parteien wirksam wird, wenn der Empfänger weiß oder wissen muss, dass das elektronische Datenschriftstück in sein Computersystem gelangt ist. Dies entspreche den herkömmlichen Kriterien, denen zufolge eine Willenserklärung dann zugegangen ist, wenn sie so in den Machtbereich des Empfängers gelangt ist, dass dieser die Möglichkeit der Kenntnisnahme hat. Bei der Bestimmung des Zugangszeitpunktes sei zwischen Unternehmern und Verbrauchern zu differenzieren, die zu unterschiedlichen Zeitpunkten davon ausgehen können, dass der Empfänger weiß oder wissen müsste, dass die Willenserklärung in seine elektronische Empfangsvorrichtung gelangt ist. Bei Verbrauchern könne nach der Verkehrssitte wie bei einem herkömmlichen Briefkasten nur davon ausgegangen werden, dass das elektronische Postfach einmal täglich überprüft wird, und zwar gewöhnlich gegen Abend. Außerhalb der Arbeitszeiten sei davon auszugehen, dass der Computer ausgeschaltet ist. Bei Unternehmern könne nur während der Betriebszeiten zwei Mal täglich, morgens und abends, erwartet werden, dass der Empfänger die Möglichkeit der Kenntnisnahme hat. ${ }^{59}$ Da Ver-

55 Dies entspricht $\$ 23$ Abs. 1 S. 2 Nr. 2 VG.

56 WANG Hongliang (Fn. 18), S. 38.

57 ZHU Qingyu (朱庆育), Allgemeine Übersicht über das Zivilrecht (民法总论), Peking 2016, S. 205.

58 Die Ausführungen Wangs beziehen sich unmittelbar auf die inhaltsgleiche Regelung des $\$ 137$ Abs. 2 S. 3 ATZR; siehe zum Verhältnis von ZGB und ATZR schon oben, Fn. 49.

59 WANG Hongliang (Fn. 18), S. 33 f. 
braucher in der VR China heutzutage häufig über Handyapps Waren bestellen und instantan über neue Nachrichten in Kenntnis gesetzt werden, erscheint zweifelhaft, ob dieses Zugangsverständnis noch zeitgemäß ist.

\section{b) Verteilung des Übermittlungsrisikos}

Auch für elektronische Willenserklärungen gilt, dass der Erklärende und der Empfänger grundsätzlich das Risiko des Verlustes und der Verzögerung bei der Übermittlung von Willenserklärungen jeweils für ihren Machtbereich übernehmen. ${ }^{60}$

(1) Zeitraum von der Abgabe der Willenserklärung bis zur Speicherung in dem Empfängersystem

Gesetzlich geregelt ist nicht, wer das Übermittlungsrisiko in dem Zeitraum von der Abgabe der elektronischen Willenserklärung bis zur Speicherung in dem Empfängersystem trägt. Das Übermittlungsrisiko umfasst den Verlust und das Abfangen der Willenserklärung während des Übermittlungsvorgangs mit der Folge, dass diese den Empfänger nicht erreicht. ${ }^{61}$ In der Literatur heißt es, in diesem Zeitraum trage der Erklärende das Übermittlungsrisiko, da er die Übermittlungsmethode wähle. Beispielhaft sei der Fall zu nennen, dass die Willenserklärung den Rechner des Empfängers nicht erreicht, es zu einer Störung der Internetverbindung, zu einem Zusammenbruch des Systems oder dazu kommt, dass die Willenserklärung durch das System gefiltert wird. Demgegenüber trage der Empfänger das Übermittlungsrisiko, wenn die Empfangsvorrichtung defekt ist oder der Empfänger die Tatsache, dass sich die Willenserklärung nicht zustellen lässt, anderweitig zu verantworten hat. Wenn der Empfänger sein elektronisches Postfach fahrlässig nicht leert und dadurch keine neuen E-Mails empfangen kann, werde dem Erklärenden das Recht eingeräumt, von dem Empfänger die unverzügliche Erklärung über den Zugang zu fordern, sodass der unterbliebene Zugang keine Verspätung darstelle. ${ }^{62}$ In dieser Situation könne vernünftigerweise nicht davon ausgegangen werden, dass der Empfänger die Möglichkeit der Kenntnisnahme hat,

60 Vgl. WANG Hongliang (Fn. 18), S. 34.

61 WANG Hongliang (Fn. 18), S. 34.

62 WANG Hongliang (Fn. 18), S. 34. 
sodass die Willenserklärung vor der Erklärung nicht zugegangen und der Erklärende nicht an sie gebunden sei; der Empfänger trage insoweit das Empfangsrisiko. ${ }^{63}$

(2) Zeitraum von der Speicherung der Willenserklärung in dem Empfängersystem bis zur Kenntnisnahme durch den Empfänger

Gesetzlich nicht geregelt ist ferner, wer das Übermittlungsrisiko trägt, wenn eine elektronische Willenserklärung in der Empfangsvorrichtung des Empfängers gespeichert wurde, sie jedoch anschließend zu einem Zeitpunkt, zu dem für gewöhnlich mit der Kenntnisnahme gerechnet werden kann, durch einen Defekt des Rechners vernichtet wird. Nach den eingangs genannten Zugangskriterien, nach denen die Zuteilung des Risikos mit dem Machtbereich der Parteien zusammenhängt, wird das Übermittlungsrisiko im Schrifttum in diesem Falle dem Empfänger zugeordnet, da sich die Willenserklärung nach der Speicherung bereits in seinem Machtbereich befinde und innerhalb eines bestimmten Zeitraums mit dessen Kenntnisnahme gerechnet werden könne. Wenn die Willenserklärung auf dem Zielrechner gespeichert wird, jedoch aufgrund eines technischen Defekts nicht zu einem Zeitpunkt, zu dem regelmäßig damit gerechnet werden kann, dass sie heruntergeladen wird, könne nach der Verkehrssitte gleichwohl vernünftigerweise mit der Kenntnisnahme durch den Empfänger gerechnet werden. Begründet liege dies wiederum in dem Umstand, dass der Empfänger selbst die entsprechenden Maßnahmen zur Möglichkeit der Kenntnisnahme von Willenserklärungen zu ergreifen habe, die in seinen Machtbereich gelangt sind. Schließlich gehe die Willenserklärung auch in dem Fall zu, dass sie auf dem Rechner des Empfängers gespeichert wird, jedoch durch ein Virus oder die versehentliche Löschung durch den Empfänger oder einen Dritten zu einem Zeitpunkt verschwindet oder unlesbar wird, in dem mit der Kenntnisnahme gerechnet werden kann, da sie den Machtbereich des Empfängers auch in diesem Fall erreicht habe. ${ }^{64}$

63 ZHU Qingyu (Fn. 57), S. 340.

64 WANG Hongliang (Fn. 18), S. 34. 


\section{Allgemeine Geschäftsbedingungen bei im Internet errichteten Verträgen}

$\mathrm{AGB}^{65}$ sind ein zumeist zentraler Bestandteil im Internet errichteter Verträge und werden in $\$ 496$ Abs. 1 ZGB legaldefiniert als Klauseln, die von einer Partei zur wiederholten Verwendung vorweg entworfen und nicht bei der Errichtung des Vertrages mit der anderen Seite ausgehandelt werden. ${ }^{66}$ So hat der Nutzer in der Regel keine Möglichkeit zur Verhandlung, sondern nur die Wahl, den einseitig gestellten AGB zuzustimmen oder diese abzulehnen. Als Beweggrund für die Nutzung von AGB wird auch in der chinesischen rechtswissenschaftlichen Literatur die Reduktion von Transaktionskosten gesehen, was Verbrauchern mittelbar durch günstigere Produkte und Dienstleistungen zugutekomme. Einher gehe mit der Nutzung von AGB durch die fehlende Möglichkeit der Einflussnahme der Vertragspartner des Verwenders während der Vertragsverhandlungen indes auch eine asymmetrische Machtverteilung, die sich zum einen in einer unzureichenden Information des Vertragspartners über Angelegenheiten der Vertragserrichtung oder Vertragsinhalte, zum anderen in dem Stellen unangemessener Klauseln äußern und so zu einer ungerechtfertigten Geltendmachung unternehmerischer Interessen führen könne. ${ }^{67}$

\section{a) Wege der Integration allgemeiner Geschäftsbedingungen bei im Internet errichteten Verträgen}

Die eingangs beschriebene Gefahr einer unangemessenen Interessenverteilung durch AGB besteht bei im Internet errichteten Verträgen in besonderer Weise, da diese in der Regel in Gestalt sogenannter Click-Wrap- und Browse-Wrap-Verträge in Erscheinung treten. Bei einem Click-Wrap-Vertrag handelt es sich um die am weitesten verbreitete Art von im Internet geschlossenen Verträgen, bei der der Nutzer sich zwecks einer effizienteren

65 格式条款; wörtlich: „Formularklauseln“; diese sind von „Geschäftsbedingungen“(交易条件) wie etwa der Gewährleistung der Qualität und angemessenen Preisen abzugrenzen, siehe hierzu Anmerkung 8 der deutschen Übersetzung des Verbraucherschutzgesetzes der VR China von Alexander Gresbrand/Madeleine Martinek/Thomas Odom et al., in: Zeitschrift für Chinesisches Recht 2014, Nr. 1, S. 69, 71.

66 Dies entspricht der Begriffsbestimmung des $₫ 39$ S. 2 VG.

67 XIA Qingfeng (夏庆锋), Regeln zur Korrektur unangemessener allgemeiner Geschäftsbedingungen in Internetverträgen (网络合同中不正当格式条款的纠正规 则), Jianghuai Tribune (江淮论坛) 2020, Nr. 2, S. 134, 134. 
Vertragsgestaltung mit sämtlichen AGB durch den Klick auf die Schaltfläche „Ich stimme zu“ einverstanden erklärt, wodurch eine rechtliche Beziehung zwischen dem Internetnutzer und dem Verwender der AGB entsteht. ${ }^{68}$ Dabei wird im Schrifttum besonders auf die Gefahr hingewiesen, dass Nutzern bei dieser Abschlussform nicht alle Vertragsinhalte angezeigt werden und die Verwender sie nicht hinreichend auf die durch die AGB entstehenden Rechte und Pflichten aufmerksam machen. Nutzern könnten auf diesem Wege in den Vertrag integrierte AGB zum Nachteil gereichen; vor allem dadurch, dass der Verwender eine für den Vertragspartner unverständliche Fachsprache verwendet, sich unklar ausdrückt, versteckte Klauseln in den Vertrag integriert oder der Inhalt zu umfangreich ist. Dies führe dazu, dass Nutzer die AGB oftmals nicht verstehen oder gar nicht erst zur Kenntnis nehmen und E-Commerce-Betreiber dies zur Verwendung unangemessener AGB nutzen. ${ }^{69}$

Sogenannte Browse-Wrap-Verträge werden ebenfalls im Internet geschlossen und auch hier werden AGB einseitig durch den Verwender gestellt. ${ }^{70}$ Anders als bei Click-Wrap-Verträgen wird bei Browse-Wrap-Verträgen für die Vertragserrichtung nicht vorausgesetzt, dass der Nutzer den durch den Verwender gestellten AGB zustimmt, indem er aktiv auf eine Schaltfläche „Ich stimme zu“ klickt; vielmehr stimmt der Nutzer den AGB durch die bloße Nutzung der Webseite zu. ${ }^{71}$ Anerkanntes Ziel auch dieser Methode ist die Förderung des E-Commerce durch die Bereitstellung einer komfortablen Methode des Vertragsschlusses. ${ }^{72}$ Befürchtet wird, dass die ohnehin schon geringe Bedeutung, die der tatsächlichen Zustimmung des Nutzers zukommt, durch das Durchbrowsen des Vertrages weiter reduziert werde und verstärkt die Gefahr unangemessener und rechtsmissbräuchlicher Klauseln bestehe. Ferner bestehe das Risiko, dass die Anerkennung von Rechtswirkungen durch Browse-Wrap-Verträge dazu führen könnte, dass Anbieter von Netzdienstleistungen Nutzer durch ein Programm dazu zwingen, auf der Seite mit den AGB zu verweilen und diesen so entgegen ihrem tatsächlichen Willen zustimmen. Da die Errichtung und Wirksamkeit von Browse-Wrap-Verträgen weder eine Mitteilung an den Empfänger

68 Cheryl B. Preston/Eli W. McCann, Unwrapping Shrinkwraps, Clickwraps, and Browsewraps: How the Law Went Wrong from Horse Traders to the Law of the Horse, in: Brigham Young University Journal of Public Law 2012, Nr. 1, S. 1, 17.

69 XIA Qingfeng (Fn. 67), S. 135.

70 Cheryl B. Preston/Eli W. McCann (Fn. 68), S. 18.

71 Cheryl B. Preston/Eli W. McCann (Fn. 68), S. 18.

72 XIA Qingfeng (Fn. 67), S. 136. 
noch dessen ausdrückliche Zustimmung voraussetze, bestehe für Nutzer ein besonders großes Risiko ungewollter vertraglicher Verpflichtungen. ${ }^{73}$

\section{b) Hinweis- und Erklärungspflichten des AGB-Verwenders}

Der chinesische Gesetzgeber hat im Lichte dieser Gefahren sowohl speziell gegenüber Verbrauchern als auch allgemeine, unabhängig von der Verbrauchereigenschaft des Vertragspartners einzuhaltende Hinweis- und Erklärungspflichten geschaffen. Die Hinweis- und Erklärungspflichten gegenüber Verbrauchern wurden im Zuge der Revision des chinesischen Verbraucherschutzrechts, deren Höhepunkt der Erlass des Verbraucherschutzgesetzes (VSG) ${ }^{74}$ in seiner Fassung vom 25.10.2013 darstellt, erweitert. ${ }^{75} \mathrm{Im}$ Vergleich zu $\$ 39$ VG sind in $\$ 26$ Abs. 1 VSG eine deutlich weitergehende Hinweis- und Erklärungspflicht geregelt, die sich im Gegensatz zu $\$ 39$ S. 1 VG nicht auf haftungsbeschränkende- und ausschließende Klauseln beschränken. Von der gegenüber allen Nutzern geltenden Regelung des $\$ 496$ Abs. 2 S. 1 ZGB unterscheidet sich diese verbraucherspezifische Regelung im Wesentlichen dadurch, dass der Inhalt der Hinweis- und Erklärungspflicht durch die beispielhafte Nennung von Inhalten, auf die sie sich bezieht, deutlich wird.

(1) Inhalt der Hinweis- und Erklärungspflichten des $\$ 496$ Abs. 2 S. 1 ZGB im Einzelnen und Beweislastverteilung

Im Einzelnen sieht $₫ 496$ Abs. 2 S. 1 ZGB wie $₫ 39$ S. 1 VG vor, dass AGBVerwender die andere Partei auf eine angemessene Art und Weise auf jene Klauseln hinweisen müssen, durch die die Haftung des Verwenders ausgeschlossen oder beschränkt wird, sowie Klauseln auf Nachfrage der anderen Partei hin erklären müssen. Insoweit kann als Orientierungshilfe vorerst weiterhin $\$ 6$ der Auslegung zum Vertragsgesetz (Teil II $)^{76}$ dienen. ${ }^{77}$ Hier-

73 XIA Qingfeng (Fn. 67), S. 136.

74 Übersetzung ins Deutsche von Alexander Gresbrand et al. (Fn. 65).

75 WANG Jianyi, Das revidierte Verbraucherschutzgesetz der Volksrepublik China, in: Recht der Internationalen Wirtschaft 2014, Nr. 5, S. 265, 267.

76 Übersetzung ins Deutsche von Knut Benjamin Pißler, in: Zeitschrift für Chinesisches Recht 2009, Nr. 3, S. 288 ff.

77 Ulrike Glück/Michael Munziger/SHI Lei, „Overview on Key Issues of the Contract Part of China's First Civil Code”, S. 9, <https://perma.cc/MFT5-4GHZ>, 
nach wird davon ausgegangen, dass der Verwender seiner Hinweispflicht auf eine angemessene Art und Weise nachgekommen ist, wenn er die haftungsbeschränkenden oder haftungsausschließenden Klauseln durch besondere Worte, Symbole oder eine besondere Schriftart bei der Vertragserrichtung hervorhebt, um die Aufmerksamkeit der anderen Vertragspartei zu erzielen, und Erklärungen jener Klauseln vornimmt, die von der Vertragspartei genannt werden. ${ }^{78}$ Erweitert hat der Gesetzgeber die Hinweisund Erklärungspflicht in $\$ 496$ Abs. 2 S. 1 ZGB gegenüber $₫ 39$ S. 1 VG insoweit, als sie sich nunmehr neben haftungsausschließenden und -beschränkenden Klauseln auch auf andere Klauseln bezieht, die in einer zur anderen Seite schwerwiegend nützlichen oder schädlichen Beziehung stehen. ${ }^{79}$ Diese offen gehaltene Formulierung des $\$ 496$ Abs. 2 S. 1 ZGB stellt eine Annäherung an $₫ 26$ Abs. 1 VSG dar und belässt dem Rechtsanwender einen Ermessensspielraum zulasten der Rechtssicherheit ${ }^{80}$, der eine flexible Reaktion im Einzelfall ermöglicht. Zur Beantwortung der Frage, welche Klauseln in einer zur anderen Seite schwerwiegend nützlichen oder schädlichen Beziehung stehen, verweist das OVG auf die in $\$ 470$ ZGB genannten Hauptvertragsinhalte. Diese fielen in der Regel unter $\$ 496$ Abs. 2 S. 1 ZGB. ${ }^{81}$

vermuten den Erlass einer ähnlichen justiziellen Auslegung zum ZGB. In seiner Kommentierung des ZGB zum Vertragsrechtsbuch verweist das OVG ebenfalls auf $\$ 6$ Abs. 2 der Auslegung zum Vertragsgesetz (Teil II) (Fn. 6), S. 246.

78 Ferner zu berücksichtigen waren in Bezug auf den konkreten Inhalt der Hinweisund Erklärungspflichten vor Inkrafttreten des ZGB eine Auslegung des OVG aus dem Jahr 2009 (最高人民法院关于适用《中华人民共和国合同法》若干问题的 解释 (二); chinesisch-englische Fassung abrufbar unter <lawinfochina.com $>$ [北大 法律英文网] / p pkulaw.cn $>$ [北大法宝], Indexnummer CLI.3.116926), zuletzt eingesehen am 5.8.2021, sowie eine große Anzahl an Vorschriften, die durch lokale Volkskongresse und durch lokale Industrie- und Handelsbehörden verabschiedet wurden, Yuanshi Bu, Das chinesische Vertragsrecht - Bestandsaufnahme und Entwicklungsperspektive, in: Zeitschrift für Europarecht, Internationales Privatrecht und Rechtsvergleichung 2014, Nr. 6, S. 261, 264. Siehe hierzu auch schon oben, Fn. 23.

79 Bereits vor Inkrafttreten des ZGB wurde in Teilen der Literatur vertreten, $\$ 39$ S. 1 VG sei so auszulegen, dass der AGB-Verwender die andere Seite angemessen auf alle Klauseln hinzuweisen habe und diese andernfalls nicht Vertragsbestandteil würden, LIU Lu (刘璐)/GAO Shengping (高圣平), Untersuchung der Regelungen über die Einbeziehung von AGB in einen Vertrag (格式条款之订入合同 规则研究), Guangxi Social Sciences (广西社会科学) 2005, Nr. 2, S. 72, 72 f.

80 So auch LI Yu (李宇), Zehn Kritikpunkte an dem Entwurf des Besonderen Teils des Zivilgesetzbuches (十评民法典分则草案), Chinese Journal of Maritime Law (中国海商法研究) 2018, Nr. 3, S.3, 6.

81 Kommentierung des OVG zum Vertragsrechtsbuch des ZGB (Fn. 6), S. 246. 
Hinsichtlich der Frage nach der Beweislastverteilung im Kontext der Hinweis- und Erklärungspflichten ist das Urteil des Höheren Volksgerichts Shanghais ${ }^{82}$ aufschlussreich. Im Einzelnen lag dem Urteil ein elektronisch abgeschlossener Vermögensverwaltungsvertrag zugrunde. Schwerpunkt der Entscheidung war, ob eine durch die Beklagte einseitig in Gestalt von AGB gestellte Schiedsklausel wirksam in den Vertrag einbezogen worden war sowie deren inhaltliche Wirksamkeit. Der Kläger machte gemäß $₫ 496$ Abs. 2 S. 2 ZGB geltend, die Klausel sei kein Vertragsbestandteil geworden, da die Beklagte ihre Erklärungspflicht nicht erfüllt habe. Da der Kläger indes nicht beweisen konnte, dass er die Erklärung wie in $\$ 496$ Abs. 2 S. 1 ZGB vorausgesetzt von der Beklagten verlangt hatte, unterstützte das Gericht die Auffassung des Klägers insoweit nicht. Die Beweislast für das Verlangen der Erklärung liegt mithin nicht bei dem AGB-Verwender. ${ }^{83}$ Neben $\$ 496$ Abs. 2 S. 1 ZGB berief sich das Gericht auf $\$ 31$ der „Erläuterungen des OVG zur Anwendung des ,Zivilprozessgesetzes der Volksrepublik China“" 84 über die Form von Gerichtsstandsklauseln bei Verbraucherverträgen. $\ 31$ besagt ebenfalls, dass nur jene Zuständigkeitsvereinbarungen unwirksam sind, auf die der Verbraucher nicht in angemessener Weise hingewiesen wurde. Das Gericht befand mithin, dass die Schiedsklausel wirksam in den Vertrag einbezogen wurde. Auch stellte es klar, dass es sich bei der Hinweispflicht des $₫ 496$ Abs. 2 S. 1 ZGB um eine „aktive Pflicht“ handele, während es sich bei der Erklärungspflicht des $\$ 496$ Abs. 2 S. 1 ZGB um eine „passive Pflicht" handele. Diese Auslegung des Gerichts stimmt mit dem Wortlaut des $\$ 496$ Abs. 2 S. 2 ZGB überein, der das „Verlangen der anderen Partei“" vorsieht, damit die Erklärungspflicht greift. ${ }^{85}$

Während die Beweislast für das Verlangen der Erklärung bei dem Vertragspartner liegt, trägt der AGB-Verwender die Beweislast für das Vorliegen der Erklärung i.S.v. $\$ 496$ Abs. 2 ZGB. In dem Urteil des Höheren Volksgerichts Shandong ${ }^{86}$ stellte sich die Frage, ob die beklagte Versicherungsgesellschaft ihre Erklärungspflicht i.S.v. $\$ 496$ Abs. 2 S. 1 ZGB erfüllt

82 Urteil des Höheren Volksgerichts Shanghai über die Hinweis- und Erklärungspflicht des AGB-Verwenders LU Jinchun gegen Shanghai Gefei Asset Management Co., Ltd., (2021) Hu Min Shen Nr. 711.

83 Das Gericht stützt sich hierbei in der Sache auf $\$ 6$ der Auslegung zum Vertragsgesetz (Teil II).

84 Übersetzung ins Deutsche von Knut Benjamin Pißler, in: Handbuch des chinesischen Zivilprozessrechts, Tübingen 2018, S. 619 ff.

85 Auch das OVG bezeichnet die Erklärungspflicht als „passive Pflicht“ (Fn. 6), S. 242.

86 Urteil des Höheren Volksgerichts Shandong über die Erklärungspflicht des AGBVerwenders Yanwo Zhenhao Fastfood Restaurant gegen CHEN Zhengao und Dongy- 
hatte. Der Kläger machte geltend, die Versicherungsgesellschaft habe nicht beweisen können, dass sie ihrer Hinweis- und Erklärungspflicht hinreichend nachgekommen sei. Das Gericht befand, dass diese Pflichten erfüllt worden seien, da der Versicherungsnehmer eine Klausel mit dem Inhalt gestempelt habe: „Der Versicherer hat den Inhalt und die Rechtsfolgen der Ausschlussklausel deutlich erklärt.“ Damit sei bewiesen, dass die Pflicht erfüllt wurde. Freilich entsteht durch die Verwendung solcher vorformulierten Klauseln zu Beweiszwecken die Gefahr, dass die Erklärungspflicht zu einer leeren Hülse verkommt.

(2) Rechte von Nutzern im Falle der Nichterfüllung der Hinweis- oder Erklärungspflichten

Vor Inkrafttreten des ZGB wurden AGB-Klauseln nicht etwa nicht einbezogen, wenn ein AGB-Verwender seiner Hinweis- oder Erklärungspflicht nicht nachkam, sondern es bestand nach $\$ 9$ der Auslegung zum Vertragsgesetz (Teil II) die Möglichkeit der Anfechtung. ${ }^{87}$ Der Grund für das Einräumen einer Anfechtungsmöglichkeit statt einer unterbliebenen Einbeziehung wurde im Schrifttum darin gesehen, dass in einem solchen Fall vom Vorliegen eines anfechtbaren Irrtums ausgegangen worden sei. ${ }^{88}$ Dem wurde entgegengehalten, dass eine Partei mangels Kenntnis der entsprechenden Punkte keinem diesbezüglichen Irrtum unterliegen und insofern auch kein Fehlurteil bilden könne. Der Irrtum über die Existenz einer Vertragsklausel sei kein Irrtum im Rechtssinne. Im Umkehrschluss aus $\mathbb{} 10$ der Auslegung zum Vertragsgesetz (Teil II) sei aber zu folgern gewesen, dass die betroffene Klausel auch dann Inhalt des Vertrages werden kann, wenn die Hinweis- und Erklärungspflicht nicht erfüllt wurden. Der Grund dafür, dass der Gesetzgeber des Vertragsgesetzes auf die Regelung einer Einbeziehungskontrolle verzichtet hatte, habe darin gelegen, dass man vermutlich von einem hinreichenden Schutz der Gegenseite durch die Inhaltskontrolle ausgegangen sei. ${ }^{89}$

ing Central Branch of Yongan Property and Casualty Insurance Co., Ltd. und Dongying Shunxing Automobile Sales Co., Ltd., (2020) Lu Min Shen Nr. 9431.

87 Yuanshi Bu (Fn. 78), S. 265. Dies legt $\$ 9$ der Auslegung zum Vertragsgesetz (Teil II) fest.

88 So TAN Zhenya (谭振亚)/HU Jian (胡建), Die Einbeziehung und Wirksamkeit von allgemeinen Geschäftsbedingungen, Journal of Yunnan Finance \& Economics University (云南财经大学学报) 2012, Nr. 1, S. 127, 142.

89 Yuanshi Bu (Fn. 78), S. 265. 
\$496 Abs. 2 S. 2 ZGB lässt eine neue Richtung erkennen: Kommt der Verwender seiner Hinweis- oder Erklärungspflicht nicht nach und nimmt die andere Partei dadurch Klauseln, an denen sie ein großes materielles Interesse hat, nicht wahr oder versteht sie nicht, kann sie gemäß $\$ 496$ Abs. 2 S. 2 ZGB geltend machen, dass diese Klauseln kein Bestandteil des Vertrages geworden sind. Erforderlich ist ab Inkrafttreten des ZGB nicht mehr die Anfechtung vor einem Volksgericht, was dem Nutzer insoweit gegenüber der Fassung des $₫ 9$ der Auslegung zum Vertragsgesetz (Teil II) zugutekommt. ${ }^{90}$ Durch die Regelung des $\$ 496$ Abs. 2 S. 2 ZGB wurde nach langem Ringen während des Gesetzgebungsverfahrens erstmals eine Einbeziehungskontrolle eingeführt. ${ }^{91}$ Abzuwarten bleibt, ob die klare Trennung zwischen Einbeziehungs- und Inhaltskontrolle fortan auch in der Rechtsprechungspraxis beherzigt wird. So ist in dem Urteil des Höheren Volksgerichts Shanghai ${ }^{92}$ zwar der Sache nach eine zweistufige Vorgehensweise des Gerichts erkennbar, indem es zunächst prüfte, ob die Hinweis- und Erklärungspflichten eingehalten wurden. Sodann wurde die Klausel einer inhaltlichen Prüfung unterzogen, bei der das Gericht feststellte, dass sie die Haftung des Verwenders nicht in unangemessener Weise ausschließe oder reduziere, die der anderen Partei erhöhe oder deren wesentliche Rechte einschränke. ${ }^{93}$ Jedoch wirkt es terminologisch unsauber, dass im Kontext

90 Glück, Ulrike/Munziger, Michael/SHI Lei (Fn. 77), S. 9; so auch das OVG in seiner Kommentierung zum Vertragsrechtsbuch des ZGB (Fn. 6), S. 8.

91 Vgl. Yuanshi Bu (Fn. 15), S. 13; zu den Voraussetzungen der wirksamen Einbeziehung von AGB nach dem ZGB siehe auch ZHAO Jin, Systematische Reform des chinesischen AGB-Rechts und seine Anwendung auf Arbeitsverträge - mit vergleichender Berücksichtigung des deutschen Rechts, 2021, S. 16 ff, abrufbar unter $<$ https://edoc.hu-berlin.de/handle/18452/23128>, zuletzt eingesehen am 5.8.2021.

92 Siehe hierzu auch oben unter II. 3. b) (1).

93 So auch das Vierte Mittlere Volksgericht Beijing, (2020) Jing 04 Min Te Nr. 780. In diesem Urteil ging es um die wirksame Einbeziehung einer Schiedsklausel in Gestalt von AGB in einen Verbraucherdarlehensvertrag sowie um deren inhaltliche Wirksamkeit. Bevor das Gericht die Wirksamkeit der Einbeziehung und die inhaltliche Wirksamkeit prüfte, stellte es zunächst fest, dass es gemäß $\mathbb{1 6}$ Schiedsgerichtsgesetz der VR China (chinesisch-englische Fassung abrufbar unter $<$ lawinfochina.com $>$ [北大法律英文网] / p pkulaw.cn $>$ [北大法宝], Indexnummer CLI.1.301395), zuletzt eingesehen am 13.8.2021, grundsätzlich möglich sei, eine Schiedsklausel in AGB festzulegen. Die Klausel sei auch wirksam in den Vertrag einbezogen worden, da der Darlehensgeber die beiden Darlehensnehmer dazu veranlasst habe, den Vertragsinhalt zur Kenntnis zu nehmen, was diese durch ihre Unterschrift auch bestätigt hätten. Hinsichtlich der inhaltlichen Wirksamkeit erkannte das Gericht, dass die Schiedsklausel keine ungültige Klausel i.S.v. $\$ \mathbb{S} 40$ VG i.V.m. 52 f. sei, da Gerichtsverfahren und Schiedsverfahren beide ihre Vorund Nachteile hätten, die alle Vertragsparteien gleichermaßen beträfen. Gegen- 
der Hinweis- und Erklärungspflichten von der „Wirksamkeit“ der Klausel die Rede ist. ${ }^{94}$

\section{Schadensersatzhaftung nach falscher Preisauszeichnung}

Nicht selten kommt es in der Praxis vor, dass Preise auf E-Commerce-Plattformen falsch ausgewiesen werden. Beteiligte des dem Urteil des Dritten Mittleren Volksgerichts Beijing ${ }^{95}$ zugrunde liegenden Rechtsstreits waren der Verbraucher LI Shanghui, Berufungskläger und Beklagter im Ausgangsverfahren, und die Beijing Century Excellence Information Technology Co., $L t d$. (Ltd.), Berufungsbeklagte und Klägerin im Ausgangsverfahren. Die Berufungsbeklagte wies auf der E-Commerce-Plattform Amazon einen Staubsauger mit Sonderfunktionen zu einem üblichen Marktpreis von 949 Yuan mit einem Kaufpreis von 94 Yuan aus. LI Shanghui bestellte drei Geräte und stimmte vorab den während des gesamten Bestellvorgangs über einen Hyperlink aufrufbaren AGB zu, in denen unter anderem klargestellt wurde, dass es sich bei den auf der Plattform präsentierten Informationen nicht um ein verbindliches Angebot, sondern lediglich um die Aufforderung zur Abgabe eines Angebots handele. In den AGB wurde ferner darauf hingewiesen, dass es zur Ausweisung falscher Preise kommen könne und die Plattform sich für den Fall, dass ein zu niedriger Preis ausgewiesen wurde, das Recht vorbehalte, den Käufer vor dem Versand zu kontaktieren oder die Bestellung zu stornieren und den Käufer darüber zu informieren. Daraufhin erhielt er eine E-Mail der $L t d$., in der diese den Eingang der Bestellung bestätigte und dabei nochmals darauf hinwies, dass die Ware nur versandt werde, solange der Vorrat reicht. Bei der Nachricht handele es sich nicht um eine Annahme, sondern lediglich um eine Empfangsbestätigung, und der Vertrag komme erst bei Versendung der Versandbestätigung zustande. Die Ware wurde jedoch nicht versandt und die von $L I$ Shanghui getätigte Zahlung an ihn zurücküberwiesen. LI Shanghui verlangte von der Ltd. die Lieferung der bestellten Ware und alternativ Zahlung

über Gerichtsverfahren hätten Schiedsverfahren zudem den Vorteil, effizient zu sein und eine endgültige Entscheidung herbeizuführen.

94 Die Vermischung von Einbeziehungs- und Inhaltskontrolle in der Rechtsprechungspraxis moniert auch HE Xuxu, Kontrolle Allgemeiner Geschäftsbedingungen (AGB) und AGB-Klauselgestaltung im Bankgeschäft, München 2011, S. $17 \mathrm{f}$.

95 Siehe Fn. 19. 
von Schadensersatz in Höhe der Preisdifferenz zwischen dem ausgewiesenen Preis und dem Marktpreis.

Das erstinstanzliche Gericht sah einen der Schwerpunkte des Falles in der Frage, ob zwischen der Ltd. und LI Shanghui ein Vertrag zustande gekommen war. Angesichts der AGB der Plattform, die den Vertragsschluss für den Zeitpunkt der Versendung der Versandbestätigung vorsahen, verneinte es dies. ${ }^{96}$ Sodann stellte das Gericht die Frage, ob die $L t d$. wegen der Verletzung einer vorvertraglichen Pflicht Schadensersatz aus $₫ 42 \mathrm{VG}^{97}$ an LI Shanghui zu zahlen habe. $\$ 42 \mathrm{VG}$ regelt die Haftung aus culpa in contrahendo. Nach $\$ 42$ Nr. 2 VG hat eine Partei Schadensersatz zu leisten, wenn sie vorsätzlich in Bezug auf die Vertragserrichtung wichtige Tatsachen verheimlicht oder zu Umständen falsche Angaben macht, sodass der anderen Partei ein Schaden entsteht. Eine Pflichtverletzung sah das Gericht in dem vorliegenden Fall darin, dass die Ltd. die Bestellung LI Shanghuis bestätigte und ihn sodann nicht über das Versehen informierte sowie seine Zahlung verhinderte, sondern die Bestellung einseitig stornierte. Aus der zwischen Onlinehändler und Verbraucher bestehenden Informationsasymmetrie folge, dass es für den Verbraucher nicht erkennbar gewesen sei, ob es sich bei dem niedrigen Preis um eine möglicherweise sogar böswillige MarketingMaßnahme handelte. Die Plattform sei überdies so zu organisieren, dass ein reibungsloser Ablauf gewährleistet wird, indem der verbleibende Lagerbestand angezeigt und verhindert wird, dass Bestellungen aufgegeben werden, wenn keine Ware mehr vorhanden ist. LI Shanghui sei die Möglichkeit des Erwerbs der Staubsauger zu einem Preis von je 94 Yuan entgangen. Beide Gerichte sprachen LI Shanghui neben der Erstattung der aufgewendeten Anwaltskosten Schadensersatz in Höhe des Differenzbetrages zwischen dem üblichen Marktpreis und dem ausgewiesenen Kaufpreis zu, was dem Erfüllungsinteresse entspricht. Dies erstaunt insofern, als der Schadensersatz aus culpa in contrabendo auch im chinesischen Recht auf den Ersatz des negativen Interesses gerichtet ist. ${ }^{98}$ Augenfällig ist zudem, dass die AGB Amazons über irrtümlich vorgenommene falsche Preisauszeichnungen keiner Wirksamkeitsprüfung unterzogen wurden.

96 Wäre das Gericht demgegenüber zu dem Ergebnis gelangt, dass ein Vertrag zwischen den Parteien zustande gekommen ist, wäre der $L t d$. lediglich die Möglichkeit der Anfechtung nach $₫ 147$ ZGB geblieben, die einen schwerwiegenden Irrtum voraussetzt.

97 Diese Regelung wurde mit leichten terminologischen Unterschieden in $\$ 500$ ZGB übernommen.

98 Siehe Fn. 11. 


\section{Die elektronische Form als Schriftform}

Gemäß $₫ 469$ Abs. 1 ZGB können Parteien einen Vertrag in Schriftform, mündlicher Form oder anderen Formen abschließen. Eine Regelung dieses Inhalts enthielt bereits $₫ 10$ S. 1 VG. In $₫ 469$ Abs. 2 ZGB wird genauer definiert, was unter der Schriftform zu verstehen ist. Hiernach ist diese eine Form, in der der Vertragsinhalt körperlich ausgedrückt werden kann, wie etwa bei Vertragsurkunden, Briefen, Telegrammen, Fernschreiben und Faxen. In $₫ 469$ Abs. 3 ZGB hat der Gesetzgeber die Fiktion für elektronische Datenschriftstücke des $\$ 4$ E-Signaturgesetz der Volksrepublik China (ESigG ${ }^{99}$ aufgenommen, die besagt, dass elektronische Datenschriftstücke in Formen wie etwa des Austausches elektronischer Daten und E-Mails, bei denen der Inhalt körperlich ausgedrückt werden kann und die jederzeit eingesehen und überprüft werden können, als Schriftform gelten. In $\$ 3$ Abs. 2 E-SigG wird ergänzend klargestellt, dass Rechtsurkunden, in denen die Parteien die Verwendung elektronischer Signaturen und elektronischer Dokumente vereinbart haben, die rechtliche Wirksamkeit nicht allein deshalb abgesprochen werden darf, weil elektronische Signaturen oder elektronische Dokumente verwendet wurden. Der Inhalt des $\$ 469$ Abs. 2 ZGB entspricht im Wesentlichen dem des $₫ 11$ VG mit dem Unterschied, dass der Gesetzgeber die in $\$ 11$ VG genannten elektronischen Datenschriftstücke in $\$ 469$ Abs. 3 ZGB eigenständig aufgeführt hat. Besteht für einen Vertrag ein Schriftformerfordernis, wie beispielsweise für den Hypothekenvertrag, $\$ 400 \mathrm{ZGB}$, oder nunmehr, wenn auch dispositiv, den Darlehensvertrag, $\mathbb{} 668$ Abs. 1 ZGB, so ist diesem mithin auch dann Genüge getan, wenn der Abschluss auf elektronischem Wege erfolgt. Ein Beispiel für einen elektronisch errichteten Darlehensvertrag liegt dem auf einen Rechtsstreit über die Eigenschaft als Darlehensnehmer folgenden Urteil des Mittleren Volksgerichts Xiamen ${ }^{100}$ zugrunde. Das Gericht stellte die Wirksamkeit des in Rede stehenden neuen papierlosen Finanzierungsproduktes für Klein- und Kleinstunternehmen fest, bei dem das Internet als Medium genutzt wird, um den gesamten Prozess der Vertragserrichtung, Darlehensvergabe und Darlehensrückforderung online durchzuführen. Eine Unterschrift auf dem Vertragsdokument ist bei diesem Modell

99 Übersetzung ins Deutsche von Simon Werthwein, in: Zeitschrift für Chinesisches Recht 2005, Nr. 2, S. $142 \mathrm{ff}$.

100 Urteil des Mittleren Volksgerichts Xiamen über die Wirksamkeit eines Darlehensvertrages ZHENG Chuanjun und Guan Tong Electronics Co., Ltd. gegen den Xiamen Xiang'an Zweig der Agricultural Bank of China Co., Ltd., (2021) Min 02 Min Zhong Nr. 899. 
nicht erforderlich, der Wille zum Vertragsschluss wird aus dem Anklicken der Schaltfläche „Zustimmen“ nach hervorgehobenem Hinweis auf die daraus resultierenden Rechtsfolgen in dem Onlinebanking-System der Bank deutlich. Der so errichtete Vertrag hat keine generell fixierte, greifbare Erscheinungsform, und nur die Vertragsparteien haben ein Recht auf Einsicht durch ihre Mobiltelefone oder ihren Computer. Während die Möglichkeit der Einsichtnahme der Parteien unabhängig voneinander der Beweisfunktion dient, darf angezweifelt werden, ob der Warnfunktion durch diese Art der Vertragserrichtung gleichermaßen Genüge getan wird wie durch eine händische Unterschrift. ${ }^{101}$

\section{Resümee}

Der chinesische Gesetzgeber hat auf die tatsächliche Entwicklung der Zunahme im Internet errichteter Verträge rechtlich reagiert. Mit Erlass der Vorschriften über den Errichtungszeitpunkt elektronischer Verträge in $\$ 491$ Abs. 2 ZGB und $\$ 49$ Abs. 1 ECG, die festlegen, dass ein elektronischer Vertrag mit Abgabe der Bestellung zustande kommt, hat der Gesetzgeber das zuvor herrschende Verständnis der Präsentation der Waren oder Dienstleistungen im Internet als invitatio ad offerendum in Frage gestellt. Letztlich stellen diese Regelungen in der Sache indes keine große Neuerung dar, da vorausgesetzt wird, dass die über das Internet bekannt gemachten Informationen die Bedingungen für ein Angebot i.S.v. 491 Abs. 2 ZGB und $\$ 49$ Abs. 1 ECG erfüllen. Dies ist nur dann gegeben, wenn sie den allgemeinen Anforderungen an ein Angebot i.S.v. $\$ 472$ ZGB gerecht werden, welche jenen entsprechen, die schon $\mathbb{\$} 14$ VG vorsah. Mit der Möglichkeit der hiervon abweichenden Vereinbarung nach $\mathbb{} 491$ Abs. 2 ZGB und $₫ 49$ Abs. 1 S. 2 ECG trägt der Gesetzgeber der Privatautonomie der Parteien Rechnung. Dafür, dass die rechtsdogmatisch und praktisch Fragen aufwerfende, bereits während des Gesetzgebungsverfahrens des ECommerce-Gesetzes sehr umstrittene Regelung des $\$ 49$ Abs. 2 ECG demgegenüber bewusst nicht in das ZGB aufgenommen wurde, sprechen gewichtige Argumente, die das OVG jedenfalls in seiner Kommentierung zum Vertragsrechtsbuch des ZGB jedoch nicht teilt. Von der Fortgeltung des $\$ 49$ Abs. 2 ECG ist daher auszugehen.

101 Vgl. hierzu auch oben unter II. 3. a) bezüglich der Gefahren sogenannter ClickWrap-Verträge. 
Elektronische Willenserklärungen werden als nicht in einem Gespräch abgegebene Willenserklärungen in dem Moment wirksam, in dem sie in das durch den Empfänger benannte Empfangssystem gelangen, dem Empfänger mithin zugehen, vgl. $\$ 137$ Abs. 2 S. 2 ZGB. Mit dem Kriterium des Zugangs in dem Moment, in dem die Willenserklärung so in den Machtbereich des Empfängers gelangt ist, dass dieser die Möglichkeit der Kenntnisnahme hat, wird auch im chinesischen Zivilrecht auf dasselbe Kriterium für den Zugang elektronischer Willenserklärungen wie für den $\mathrm{Zu}$ gang herkömmlicher Willenserklärungen rekurriert. Besonders hervorzuheben ist die Neuerung des $₫ 137$ Abs. 2 S. 3 ZGB gegenüber $₫ 16$ S. 2 VG zur Regelung der Situation, dass ein Empfänger über mehrere Empfangssysteme zum Erhalt elektronischer Willenserklärungen verfügt und keines davon benannt hat. Abgestellt wird für den Zugang nunmehr nicht auf den Zeitpunkt, in dem die Willenserklärung erstmals in irgendein Computersystem des Empfängers gelangt, sondern auf den Zeitpunkt, ab dem der Empfänger hiervon weiß oder wissen müsste. Dies entspricht den Kriterien für den Zugang herkömmlicher Willenserklärungen. Angesichts der dem Empfänger so verbleibenden Entscheidungsfreiheit darüber, bestimmte, durch ihn eingerichtete Empfangssysteme nicht zu Zwecken der Vertragserrichtung zu nutzen, ist dies zu begrüßen. Die Gefahr der Übermittlung ist ebenfalls an den verschiedenen Machtbereichen orientiert und stellt insofern eine ausgeglichene Risikoverteilung dar.

Beweggrund für die Verwendung von AGB ist auch im chinesischen $\mathrm{Zi}$ vilrecht die Steigerung der Effizienz des Vertragsschlusses. Vertragspartnern von AGB-Verwendern kommt dies durch die Reduktion von Transaktionskosten einerseits zugute, andererseits befinden sie sich durch das so entstehende informationelle und technische Machtgefälle in einer schwächeren und insofern besonders schutzwürdigen Position. Dies gilt für Browse-Wrap- sowie für die weiter verbreiteten Click-Wrap-Verträge. Der chinesische Gesetzgeber hat hierauf sowohl durch die Schaffung der gegenüber Verbrauchern geltenden Hinweis- und Erklärungspflicht des $\mathbb{2 6}$ VSG als auch im Zuge des Erlasses des ZGB reagiert, indem er die bereits zuvor in $\$ 39$ S. 1 VG geregelte Hinweis- und Erklärungspflicht in $\$ 496$ Abs. 2 S. 1 ZGB aufgenommen und erweitert hat. Die generalklauselartige Erweiterung dieser Pflichten auf jene Klauseln, die mit materiellen Interessen der Vertragspartei in Verbindung stehen, zeugt von einem Gespür des Gesetzgebers für die Vielfalt der für den Vertragspartner potenziell bedeutsamen Klauseln, die nicht auf haftungsbeschränkende und -ausschließende Klauseln begrenzt und einer abschließenden Regelung unzugänglich sind. Ermöglicht wird eine flexible Handhabung im Einzelfall, die durch den so geschaffenen Ermessensspielraum auf Kosten der Rechtssicherheit geht. 
Hinsichtlich der Rechte von Internetnutzern und Verbrauchern für den Fall der Nichterfüllung der zuvor genannten Pflichten ist die durch $₫ 496$ Abs. 2 S. 2 ZGB geschaffene, dem chinesischen Recht zuvor noch fremde Einbeziehungskontrolle besonders hervorhebungswürdig. Prozessual stellt sie eine Erleichterung für Nutzer dar, da sie sich auf die Unwirksamkeit von Klauseln berufen können, ohne dies zuvor gerichtlich geltend gemacht zu haben.

Für den praktisch relevanten Fall, dass Preise auf E-Commerce-Plattformen falsch ausgewiesen werden, stellt sich die Frage, ob bereits ein Vertrag geschlossen wurde, der sodann angefochten werden kann. Ist dem nicht so, droht eine Haftung aus culpa in contrahendo, wenn der Verbraucher nicht über das Versehen in Kenntnis gesetzt, sondern die Bestellung einseitig durch den auf der Plattform tätigen Betreiber storniert wird.

Mit der gesetzlichen Fiktion in $\$ 469$ Abs. 3 ZGB darüber, dass elektronische Datenschriftstücke als Schriftform gelten, hat der Gesetzgeber zuvor bereits im VG und E-SigG existierende Regelungen in das ZGB aufgenommen und ihre Geltung damit bekräftigt. Die Regelung kann als Abwägungsentscheidung des Gesetzgebers zugunsten der Möglichkeit einer flexiblen und effizienten Vertragserrichtung verstanden werden, welche die Bedeutung der dem Schriftformerfordernis inhärenten Warnfunktion schmälert.

Insgesamt ist festzuhalten, dass die Vertragserrichtung im Internet in der VR China seit Erlass des ZGB eine rechtliche Aufwertung erfahren hat, deren Ausmaß sich angesichts der Übernahme einiger bereits zuvor existierender Regelungen jedoch in Grenzen hält. 
